Produto \& Produção, vol. 11, n. 1, p. 11 - 21, fev. 2010

\title{
Apreciação Técnica de Modelo de Esfigmomanômetros Digitais
}

Ronaldo N. Azeredo rnazeredo@inmetro.gov.br

Renata A. R. Pereira rapereira@inmetro.gov.br

Flavio W. Sant'Ana fwsantana@inmetro.gov.br

José A. G. Neto janeto@inmetro.gov.br

Instituto Nacional de Metrologia, Normalização e Qualidade Industrial - Inmetro Diretoria de Metrologia Legal - Dimel I Div. Instr. Medição no Âmb. Saúde e Meio Ambiente - Disma

\section{RESUMO}

Será apresentado um panorama dos resultados obtidos nos processos de apreciação técnica de modelo de esfigmomanômetros digitais, realizados pela Diretoria de Metrologia Legal do Inmetro entre janeiro de 2007 e dezembro de 2008.

Palavras-chave: Metrologia Legal, regulamentação, esfigmomanômetros.

\section{INTRODUÇÃO}

\subsection{Controle Metrológico Legal}

Denomina-se controle metrológico legal o conjunto de atividades de metrologia legal que visam à garantia metrológica, compreendendo o controle legal dos instrumentos de medição, a supervisão metrológica e a perícia metrológica (INMETRO, 2005a). Na área da saúde, a atividade de maior destaque é o controle legal dos instrumentos de medição.

Todo instrumento de medição sob o controle legal deve ser submetido à apreciação técnica de modelo (ATM) antes de ser colocado no mercado. Esta atividade é responsabilidade da Diretoria de Metrologia Legal do Inmetro (Dimel) e compreende o exame e ensaio sistemáticos do desempenho de um ou vários exemplares de um modelo identificado de um instrumento de medição, em relação 
às exigências documentadas, a fim de determinar se o modelo pode ou não ser aprovado (INMETRO, 2005a).

As exigências que os instrumentos devem cumprir estão determinadas no Regulamento Técnico Metrológico (RTM), específico para cada tipo de instrumento de medição. Normalmente o RTM é desenvolvido com base numa recomendação da Organização Internacional de Metrologia Legal (OIML). Se não houver recomendação da OIML, os requisitos do RTM devem ser baseados em outro documento de aceitação internacional como, por exemplo, os publicados pela International Organization for Standardization (ISO) ou pela International Electrotechnical Commission (IEC).

O processo de ATM compreende uma avaliação de documentos (manuais de instruções, desenhos, etc.), uma inspeção visual (inscrições, uso correto das unidades de medida, legibilidade das indicações, etc.) e ensaios de desempenho.

Quando o instrumento de medição obtém êxito no processo de ATM, considera-se que o seu modelo foi aprovado. A comunicação dessa decisão é feita através da publicação de uma Portaria de Aprovação de Modelo.

A etapa seguinte do controle legal é a verificação, que é o procedimento que inclui o exame e a marcação e/ou a emissão de um certificado de verificação e que constata e confirma que o instrumento de medição satisfaz às exigências regulamentares (INMETRO, 2005a). Os requisitos a serem atendidos estão determinados no mesmo RTM utilizado na ATM.

Todo exemplar de instrumento novo deve ser submetido à verificação inicial antes de ser vendido ao seu usuário final, quando são realizados alguns ensaios básicos para confirmar que o exemplar mantém as características do modelo que foi aprovado. A verificação inicial pode ser individual (todas as unidades) ou por amostragem.

Se o exemplar for aprovado na verificação, ele recebe uma marca de selagem (para evitar abertura não-autorizada, quando for o caso) e uma marca de verificação. Depois disso ele pode ser comercializado e colocado em uso.

As verificações subsequentes também fazem parte do controle legal dos instrumentos de medição. Elas podem ser feitas em intervalos de tempo prédeterminados (verificação periódica) ou sob solicitação do detentor do instrumento (verificação voluntária).

\subsection{Medição da Pressão Arterial}

A medicina depende da medição de diversos parâmetros fisiológicos para a definição de diagnósticos e terapias. Remonta à Grécia Antiga (300 a.C) a primeira descrição técnica da pulsação e sua relação com a saúde humana (INTROCASO, 1996).

Durante os séculos seguintes foram realizados diversos experimentos sobre a medição da pulsação, permitindo o aprofundamento dos estudos sobre circulação sanguínea. Porém, somente no século XVII a medicina conseguiu perceber a existência da pressão sanguínea, graças a um experimento com animais feito pelo reverendo inglês Stephen Hales, a quem se atribui a realização da primeira medição invasiva da pressão arterial, representada na Figura 1 (INTROCASO, 1996). 


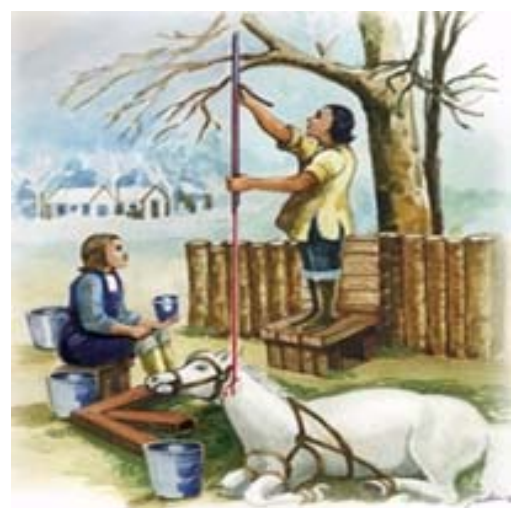

Figura 1 - Representação da primeira medição invasiva da pressão arterial

Diversos experimentos e instrumentos de medição foram desenvolvidos nos anos seguintes até que, em 1896, Scipione Riva-Rocci apresentou a concepção do instrumento que conhecemos hoje, o esfigmomanômetro. Esse instrumento simples e de fácil manuseio marcou o fim da era das pesquisas de um método clínico para a medição não-invasiva da pressão arterial humana (INTROCASO, 1996).

Atualmente os esfigmomanômetros baseados na concepção de Riva-Rocci dividem-se em dois tipos: aneróides e de coluna de líquido manométrico.

No esfigmomanômetro do tipo aneróide, o ar é utilizado como o fluido manométrico que irá deformar o sensor elástico do instrumento e causar o deslocamento do ponteiro sobre a escala, indicando a pressão arterial (INMETRO, 2005b). No esfigmomanômetro de coluna de líquido manométrico, a pressão é indicada em função do deslocamento de uma coluna de líquido manométrico num tubo transparente graduado (INMETRO, 2005b). Na maioria dos casos o líquido utilizado é o mercúrio.

A rápida evolução da eletrônica e sua grande aplicabilidade nos instrumentos de medição fez surgir no final da década de 1970 o esfigmomanômetro digital (INTROCASO, 1996).

Para o usuário do instrumento, talvez a principal diferença entre os esfigmomanômetro mecânicos e os digitais seja a facilidade de uso. No instrumento digital, o processo de detecção das pressões sistólica (máxima) e diastólica (mínima) é feito pelo próprio instrumento, dispensando a presença de um profissional treinado para a obtenção dos resultados.

Devido a essa facilidade de uso, o esfigmomanômetro digital torna-se uma atraente opção, principalmente para pessoas que precisam monitorar constantemente sua própria pressão arterial. Porém, uma vez que a medição é feita totalmente pelo próprio instrumento, muitas vezes surge a seguinte questão: "posso confiar nos resultados apresentados pelo esfigmomanômetro digital?"

Buscando responder a essa pergunta e proteger a saúde do cidadão, o Inmetro iniciou em 2006 o controle metrológico legal dos esfigmomanômetros digitais, cujos detalhamentos e resultados são apresentados a seguir.

\section{OBJETIVO}

Apresentar um panorama dos resultados obtidos nos processos de apreciação técnica de modelo (ATM) de esfigmomanômetros digitais, realizados pela Diretoria de Metrologia Legal do Inmetro entre janeiro de 2007 e dezembro de 2008. 
Para isso, será detalhado o controle metrológico legal dos esfigmomanômetros digitais, destacando-se os seguintes pontos:

- Principais características técnicas e de funcionamento;

- Regulamentação aplicável;

- Estrutura da apreciação técnica de modelo;

- Principais características dos ensaios realizados;

- Caracterização dos modelos de esfigmomanômetro digital disponíveis à sociedade.

\section{PRINCIPAIS CARACTERÍSTICAS TÉCNICAS}

Um esfigmomanômetro digital possui basicamente os seguintes componentes:

- Braçadeira: possui uma parte inflável (manguito), sendo aplicada ao braço, coxa ou punho do paciente para obstruir o fluxo de sangue na artéria;

- Bomba de ar: utilizada para insuflar o manguito, pode ser elétrica ou manual;

- Válvulas de exaustão: servem para controlar a saída do ar durante o esvaziamento do manguito;

- Manômetro: responsável por indicar a pressão aplicada no manguito e realizar a determinação das pressões arteriais máxima e mínima.

Quanto ao seu funcionamento, podem ser semi-automáticos ou automáticos. Os esfigmomanômetros do tipo semi-automático (Figura 2) utilizam uma bomba de ar manual operada pelo próprio paciente e podem medir a pressão arterial no braço ou na coxa. Após elevar a pressão até um determinado valor (inflação), o paciente para de bombear e o próprio esfigmomanômetro passa a controlar o esvaziamento do manguito (deflação). Após finalizar a medição, alguns modelos realizam automaticamente a deflação rápida, enquanto outros precisam que o paciente acione uma válvula para retirar o restante de ar do manguito.
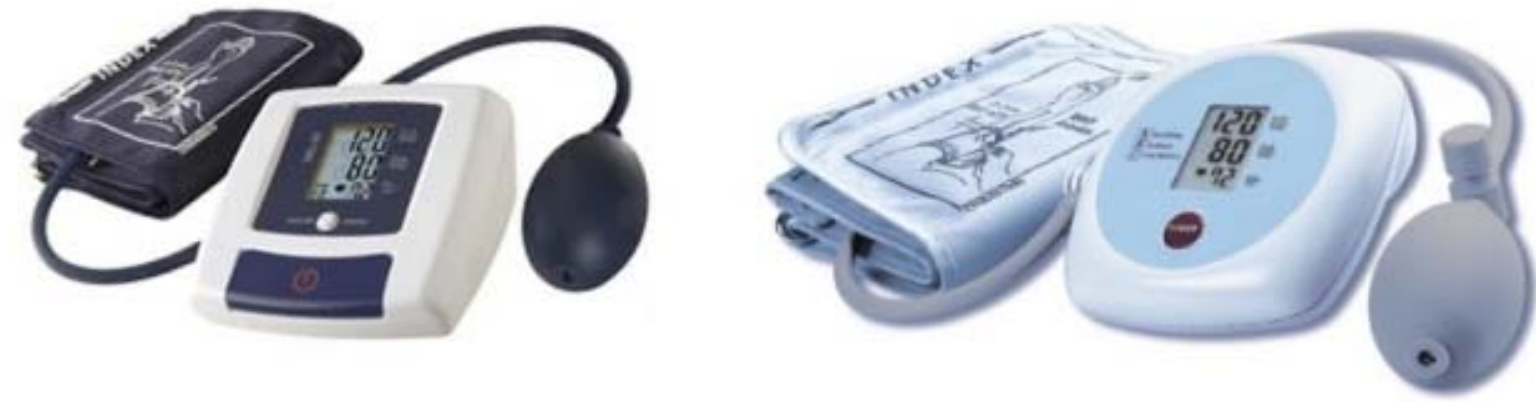

Figura 2 - Exemplos de esfigmomanômetros digitais semi-automáticos

Nos esfigmomanômetros do tipo automático, por sua vez, o paciente precisa apenas acionar o botão para início da medição e o instrumento realiza sozinho todas as etapas para medição da pressão. Eles podem medir a pressão no braço/coxa (Figura 3, instrumentos à esquerda) ou no punho do paciente (Figura 3, instrumentos à direita). 

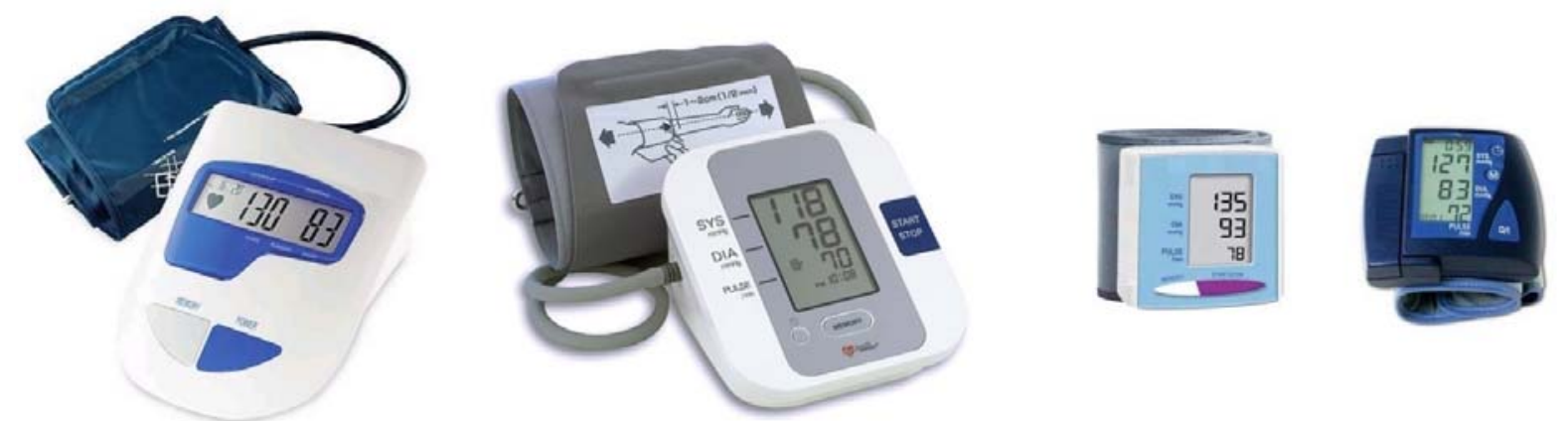

Figura 3 - Exemplos de esfigmomanômetros digitais automáticos

Os esfigmomanômetros digitais podem utilizar dois métodos de medição: auscultatório ou oscilométrico.

O método auscultatório utiliza os sons de Korotkoff, que são gerados pela artéria ocluída enquanto a pressão é lentamente diminuída. O primeiro som gerado coincide com a pressão sistólica, enquanto que o último coincide com a pressão diastólica (OIML, 2002). Para captar os sons de Korotkoff, a braçadeira dos esfigmomanômetros digitais que utilizam o método auscultatório possui um pequeno microfone acoplado. A determinação da pressão arterial pode ser feita apenas durante a deflação.

No método oscilométrico, durante a inflação e a deflação ocorrem pequenas mudanças na pressão do manguito (oscilações) como resultado dos pulsos da pressão arterial. Essas oscilações, que primeiro aumentam e depois diminuem, são detectadas e armazenadas junto com os valores de pressão correspondentes. Através de um algoritmo matemático adequado, esses valores são utilizados para calcular as pressões sistólica, diastólica e média. A determinação da pressão arterial pode ser feita tanto na inflação quanto na deflação (OIML, 2002).

Além da pressão arterial, os esfigmomanômetros digitais medem também os batimentos cardíacos. Alguns modelos possuem ainda funções específicas, como memória para armazenar os resultados de medição, detecção de arritmias cardíacas ou possibilidade de conexão com o computador (para transferência dos resultados de medição) ou com impressora específica.

\section{APRECIAÇÃO TÉCNICA DE MODELO}

\subsection{Regulamentação Aplicável}

A OIML possui a Recomendação Internacional R16-2 - Non-invasive automated sphygmomanometers. A edição atual desse documento é de 2002 e sua revisão está prevista para o final de 2009 , visando compatibilizá-la com os requisitos da norma ISO sobre esfigmomanômetros que será publicada em breve.

Em 2006 foi publicada a Portaria Inmetro $n^{\circ} 336$, aprovando o primeiro RTM sobre esfigmomanômetros digitais, desenvolvido com base na OIML R16-2. Durante sua implementação foram detectadas diversas oportunidades de melhoria para torná-lo mais adequado à realidade brasileira, que culminaram numa revisão total do RTM, publicada através da Portaria Inmetro no 096/2008.

No RTM estão estabelecidas as condições mínimas que os esfigmomanômetros digitais devem atender para que sejam aprovados pelo Inmetro. 


\subsection{Estrutura da ATM}

A ATM dos esfigmomanômetros digitais é realizada através do exame de documentos e de uma amostra com 03 exemplares (INMETRO, 2008a).

Modelos estruturalmente idênticos podem ser agrupados em um único processo de apreciação, sob o conceito de família de instrumentos. São admitidas pequenas diferenças, como aparência externa ou funções extras (por exemplo, diferente capacidade de memória).

No Exame da Documentação é verificado se a documentação está completa e se ela esclarece e define as especificações técnicas e as características construtivas e metrológicas do esfigmomanômetro.

Em seguida, a amostra é submetida ao Exame Geral, que consiste em verificar a conformidade do esfigmomanômetro com a documentação apresentada, realizando uma avaliação inicial quanto às suas características técnicas e metrológicas, inscrições, indicação dos resultados e locais de selagem, conforme as exigências regulamentares aplicáveis.

As não-conformidades detectadas nas duas primeiras etapas podem ser corrigidas pelo fabricante/importador em até 60 dias, sem que o processo de apreciação seja encerrado (INMETRO, 2008b).

A última etapa são os Ensaios de Desempenho, divididos em Básicos (8 ensaios) e Complementares (10 ensaios) (INMETRO, 2008a). Dependendo do ensaio, a referência utilizada pode ser um manômetro padrão (resolução melhor ou igual a $0,2 \mathrm{mmHg} 1$ e incerteza máxima de $0,8 \mathrm{mmHg}$ ) ou um equipamento comumente chamado de "simulador de paciente", usado para testar a repetitividade do esfigmomanômetro (gera pulsos oscilométricos que são interpretados como pressão arterial).

Para que o modelo seja considerado aprovado num determinado ensaio, é necessário que todos os exemplares da amostra obtenham êxito nesse ensaio. Caso qualquer um dos exemplares apresente não-conformidade, toda a amostra é considerada reprovada e o processo é encerrado (INMETRO, 2008b).

Com base no Artigo $4^{\circ}$ da Portaria Inmetro $n^{\circ}$ 096/2008, os modelos que obtiveram êxito nos ensaios básicos receberam uma Portaria de Aprovação de Modelo (PAM) provisória, válida até 31/12/08. Para obter a PAM definitiva foi necessário obter êxito em todas as etapas da ATM. Atualmente são realizados todos os ensaios em uma única etapa e, em caso de aprovação, é emitida a PAM definitiva.

\subsection{Ensaios de ATM}

Visando garantir a confiabilidade dos resultados obtidos, os ensaios buscam simular diversas condições a que o esfigmomanômetro pode ser submetido durante

\footnotetext{
${ }^{1}$ Apesar de não ser a unidade de pressão prevista, o Sistema Internacional de Unidades (SI) aceita o uso da unidade milímetro de mercúrio $(\mathrm{mmHg})$ na medição de pressão arterial, dentre outras aplicações específicas.
} 
o uso. A seguir serão apresentadas as principais características dos ensaios de ATM de esfigmomanômetros digitais (INMETRO, 2008a).

- Determinação do erro de indicação: consiste em realizar medições estáticas de pressão no manômetro do instrumento na faixa de medição informada pelo fabricante, em um ciclo crescente (carga) seguido de um ciclo decrescente (descarga). O modelo deve atender ao erro máximo admissível de \pm 3 $\mathrm{mmHg}$;

- Determinação da regulagem da válvula de deflação: aplicável somente aos modelos que realizam a medição pelo método auscultatório, consiste em verificar se o instrumento permite o ajuste da taxa de redução de pressão para 2 $\mathrm{mmHg} / \mathrm{s}$ a $3 \mathrm{mmHg} / \mathrm{s}$;

- Determinação da deflação rápida: consiste em verificar se o modelo permite o rápido esvaziamento do manguito, o que pode ser necessário em uma situação de emergência durante a medição;

- Determinação do escapamento de ar: verifica-se a capacidade do modelo manter a pressão durante $5 \mathrm{~min}$;

- Choque mecânico: o modelo deve resistir a pequenas quedas $(5 \mathrm{~cm}$ de altura) sobre uma superfície rígida, situação comum durante o uso normal;

- Ajuste de zero: consiste em verificar se o ajuste do zero é realizado automaticamente;

- Deriva da indicação de pressão: aplicável somente aos modelos que realizam ajuste de zero apenas ao serem ligados, consiste em verificar se o modelo se desliga automaticamente antes que a deriva da indicação exceda 1 $\mathrm{mmHg}$;

- Interrupção imediata da medição: consiste em verificar se é possível interromper a medição da pressão arterial, a qualquer momento, através do acionamento de uma chave ou botão, levando o modelo a realizar uma exaustão rápida;

- Fadiga: consiste em verificar se, após ser submetido a 10.000 ciclos de pressão, as indicações do modelo não apresentam alteração maior que $3 \mathrm{mmHg}$;

- Portas de entrada e saída de sinais: aplicável somente aos modelos que possuem portas de comunicação, consiste em verificar se acessórios encaixados incorretamente ou defeituosos não afetam os resultados de medição;

- Fonte de alimentação interna: é aplicável somente aos modelos que funcionam com pilhas. Consiste em comparar as indicações do modelo na sua tensão nominal com as indicações na tensão mínima na qual o dispositivo indicador ainda apresenta resultados, acrescida de 0,1 V;

- Fonte de alimentação externa: é aplicável somente aos modelos que funcionam em corrente alternada ou que possuem entrada para eliminador de pilhas. Consiste em comparar as indicações do esfigmomanômetro na sua tensão nominal com as indicações sob tensões mínimas de funcionamento;

- Determinação do erro em função da variação da temperatura: verificase a divergência entre as indicações do esfigmomanômetro nas temperaturas de $10^{\circ} \mathrm{C}, 20^{\circ} \mathrm{C}$ e $40^{\circ} \mathrm{C}$; 
- Armazenamento: verifica-se a divergência entre as indicações do esfigmomanômetro antes e após ser submetido a condições ambientais que simulam armazenamento;

- Descargas eletrostáticas: consiste em verificar se descargas eletrostáticas por contato e pelo ar acarretam erro de indicação maior que o admissível;

- Campos eletromagnéticos radiados: consiste em verificar se campos eletromagnéticos similares aos gerados por aparelhos como, por exemplo, telefone celulares, acarretam erro de indicação maior que o admissível;

- Transientes elétricos: aplicável somente aos modelos que funcionam em corrente alternada, consiste em verificar a imunidade a transientes nas linhas de alimentação e de dados/controle;

- Redução da tensão de alimentação por curto intervalo de tempo: aplicável somente aos modelos que funcionam em corrente alternada, consiste em verificar o comportamento do esfigmomanômetro quando submetido a reduções na tensão de alimentação.

Como se pode perceber, diversos ensaios são aplicáveis somente a modelos de esfigmomanômetro digital que possuam determinadas características técnicas, fazendo com que a quantidade total de ensaios a serem realizados seja variável.

\section{RESULTADOS}

A seguir serão apresentadas diversas informações sobre os processos de ATM que foram protocolados no Inmetro entre janeiro de 2007 e dezembro de 2008.

\subsection{Quantidade Total de Processos}

Foram protocolados 51 processos de ATM, relativos a 72 modelos de esfigmomanômetros digitais. Desse total, em 12 processos (14 modelos) houve desistência por parte das empresas solicitantes. Dos 39 processos restantes, 34 foram concluídos (50 modelos) e 5 continuam em andamento2 (8 modelos, todos já testados).

\subsection{Características Técnicas dos Modelos}

A Figura 4 mostra a distribuição dos 58 modelos testados de acordo com seu modo de funcionamento, onde se pode perceber forte predominância de modelos para medição da pressão arterial no punho (53\% do total).

\footnotetext{
${ }^{2}$ Dados relativos a abril/2009.
} 


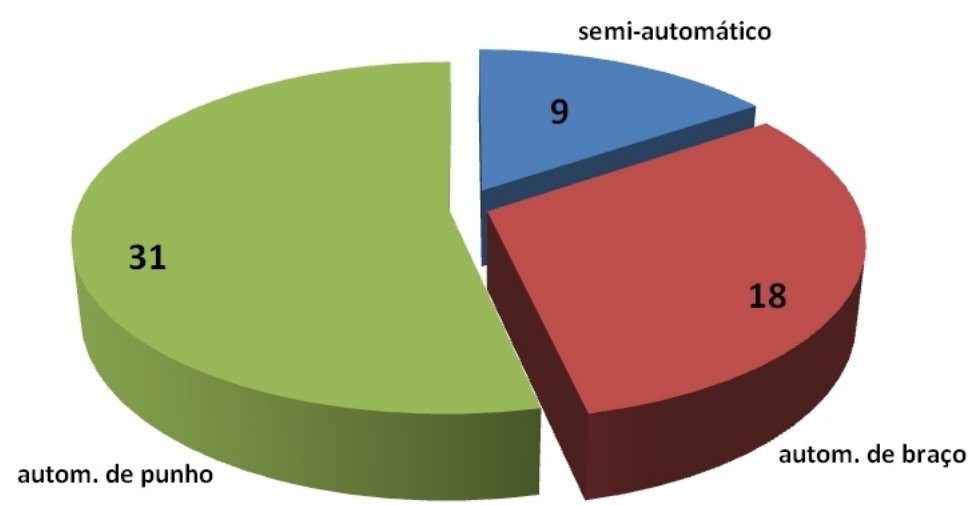

Figura 4 - Distribuição dos modelos por modo de funcionamento

Quanto ao método de medição, todos os modelos utilizam o método oscilométrico.

\subsection{Origem dos Modelos}

Todas as 11 empresas que solicitaram ATM de esfigmomanômetros digitais são importadoras, comercializando esse instrumento no Brasil sob 13 marcas diferentes.

Os modelos são fabricados por 13 empresas no exterior. A Figura 5 mostra a distribuição dos modelos de acordo com o país de origem.

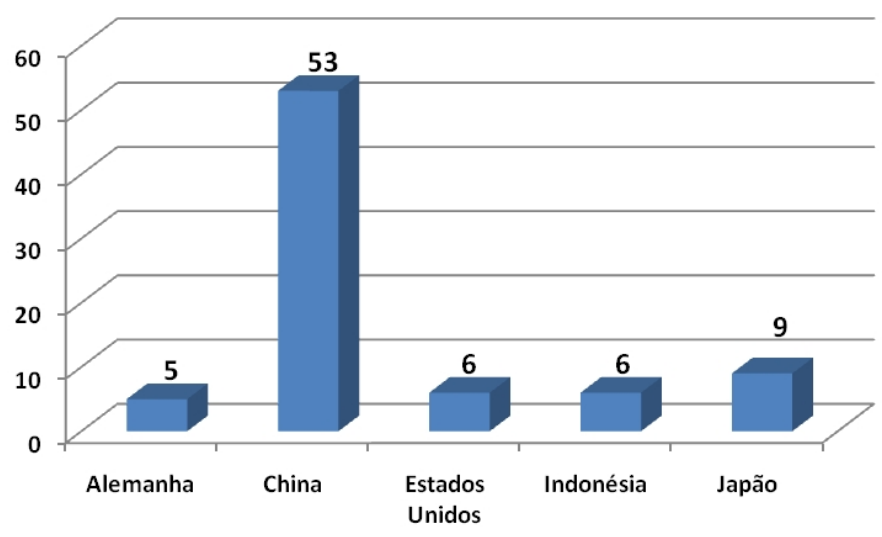

Figura 5. Distribuição dos modelos por país de origem

Cabe ressaltar que 9 modelos podem ser fabricados em mais de um país, fazendo com que a quantidade de modelos listados na Figura 5 ultrapasse 58 modelos.

\subsection{Ensaios}

Todos os 58 modelos testados obtiveram êxito nos Ensaios Básicos de Desempenho, recebendo uma PAM provisória que foi válida até 31/12/2008. Porém, 9 modelos foram reprovados em Ensaios Complementares, conforme listado a seguir: 
5 modelos no ensaio de Determinação do Erro em Função da Variação da Temperatura; e

4 modelos no ensaio de Campos Eletromagnéticos Radiados.

Dentre os modelos reprovados, 2 realizaram as correções necessárias, foram submetidos à ATM em novos processos e obtiveram êxito.

Até final de julho de 2009, foram emitidas 28 PAM definitivas, aprovando 41 modelos de esfigmomanômetros digitais. Somando-se os 8 modelos que já foram testados e ainda não receberam a PAM definitiva devido à pendências na documentação, totaliza-se 49 modelos. O índice de modelos aprovados (85\%) pode ser considerado muito bom, pois é comum que na implantação de uma nova regulamentação esse índice fique em torno de $60 \%$.

\title{
6 CONCLUSÃO
}

A atividade iniciada pelo Inmetro gera claro benefício à sociedade, uma vez que confere credibilidade aos resultados de medição obtidos com os esfigmomanômetros digitais à venda no país, retirando do mercado os modelos que não cumprem os requisitos exigidos pelo RTM.

Além disso, ao definir padrões mínimos de qualidade para os instrumentos que serão comercializados, o Brasil se posiciona perante o comércio internacional e deixa de ser visto como um país no qual é possível comercializar produtos de qualidade duvidosa.

A regulamentação também é benéfica para os próprios fabricantes e importadores, pois a marca do Inmetro agrega valor ao produto e melhora sua aceitação junto ao consumidor, diminuindo os questionamentos sobre a confiabilidade dos resultados das medições dos esfigmomanômetros digitais.

\begin{abstract}
This paper presents a result's overview of the pattern approval processes performed at Inmetro's Legal Metrology Directorate between January '07 and December '08.
\end{abstract}

Keywords: Legal Metrology, regulation, sphygmomanometers.

\section{Agradecimentos}

Agradecemos a todos que colaboraram no desenvolvimento das atividades citadas no presente artigo. 


\section{REFERÊNCIAS}

INMETRO. Portaria Inmetro $n^{\circ}$ 163/2005 - Vocabulário Internacional de Termos de Metrologia Legal. 2005a. Disponível em: http://www.inmetro.gov.br.

Portaria Inmetro $n^{\circ}$ 153/2005 - Regulamento Técnico Metrológico sobre esfigmomanômetros mecânicos. 2005b. Disponível em: http://www.inmetro.gov.br.

. Portaria Inmetro $n^{\circ}$ 096/2008 - Regulamento Técnico Metrológico sobre esfigmomanômetros digitais. 2008a. Disponível em: http://www.inmetro.gov.br.

. NIE-Dimel-067 - Diretrizes para ensaios no processo de apreciação técnica de modelo. Rev. 03, Jun/2008b. Disponível em: http://www.inmetro.gov.br

INTROCASO, Luiz. História da Medida da Pressão Arterial - 100 Anos do Esfigmomanômetro. Arquivos Brasileiros de Cardiologia Online, Vol. 67, nº 5, 1996. Disponível em: http://publicacoes.cardiol.br/abc.

OIML. International Recommendation R16-2: Non-invasive automated sphygmomanometers. 2002. Disponível em: http://www.oiml.org. 